\title{
Analytical Solution of Thermal Radiation and Chemical Reaction Effects on Unsteady MHD Convection through Porous Media with Heat Source/Sink
}

\author{
Abdel-Nasser A. Osman, ${ }^{1}$ S. M. Abo-Dahab, ${ }^{1,2}$ \\ and R. A. Mohamed ${ }^{1}$ \\ ${ }^{1}$ Mathematics Department, Faculty of Science, SVU, Qena 83523, Egypt \\ ${ }^{2}$ Department of Mathematics, Faculty of Science, Taif University, Taif 21974, Saudi Arabia \\ Correspondence should be addressed to S. M. Abo-Dahab, sdahb@yahoo.com
}

Received 29 March 2011; Accepted 27 May 2011

Academic Editor: Moran Wang

Copyright ( 2011 Abdel-Nasser A. Osman et al. This is an open access article distributed under the Creative Commons Attribution License, which permits unrestricted use, distribution, and reproduction in any medium, provided the original work is properly cited.

This paper analytically studies the thermal radiation and chemical reaction effect on unsteady MHD convection through a porous medium bounded by an infinite vertical plate. The fluid considered here is a gray, absorbing-emitting but nonscattering medium, and the Rosseland approximation is used to describe the radiative heat flux in the energy equation. The dimensionless governing equations are solved using Laplace transform technique. The resulting velocity, temperature and concentration profiles as well as the skin-friction, rate of heat, and mass transfer are shown graphically for different values of physical parameters involved.

\section{Introduction}

Thermal radiation of a gray fluid which is emitting and absorbing radiation in a nonscattering medium has been investigated by Ali et al. [1], Ibrahim and Hady [2], Mansour [3], Hossain et al. [4, 5], Raptis and Perdikis [6], Makinde [7], and Abdus-Sattar and Hamid Kalim [8]. All these studies have investigated the unsteady flow in a nonporous medium. From the previous literature survey about unsteady fluid flow, we observe that few papers were done in a porous medium. The radiative flows of an electrically conducting fluid with high temperature in the presence of a magnetic field are encountered in electrical power generation, astrophysical flows, solar power technology, space vehicle, nuclear engineering application, and other industrial areas. The analytical solution of unsteady MHD laminar convective flow with thermal radiation of a conducting fluid with variable properties through a porous medium in the presence of chemical reaction and heat source or sink has not been investigated. 
Combined heat and mass transfer problems with chemical reaction are of importance in many processes and have, therefore, received a considerable amount of attention in the recent years. In processes such as drying, evaporation at the surface of a water body, energy transfer in a wet cooling tower and the flow in a desert cooler, heat and mass transfer occur simultaneously. Possible applications of this type of flow can be found in many industries, for example, in the electric power industry, among the methods of generating electric power is one in which electrical energy is extracted directly from a moving conducting fluid. Many practical diffusion operations involve the molecular diffusion of a species in the presence of chemical reaction within or at the boundary. There are two types of reactions. A homogeneous reaction is one that occurs uniformly throughout a give phase. The species generation in a homogeneous reaction is analogous to internal source of heat generation. In contrast, a heterogeneous reaction takes place in a restricted region or within the boundary of a phase. It can therefore be treated as a boundary condition similar to the constant heat flux condition in heat transfer. Combined heat and mass transfer with chemical reaction in geometric with and without porous media has been studied by others [9-19].

This paper deals with the study of thermal radiation and chemical reaction effects on the unsteady MHD convection through a porous medium bounded by an infinite vertical plate with heat source/sink. The governing equations are solved by Laplace transform technique. The results are obtained for velocity, temperature, concentration, skin-friction, rate of heat and mass transfer. The effects of various material parameters are discussed on flow variables and presented by graphs.

\section{Formulation of the Problem}

Consider the unsteady free convection flow of an incompressible viscous fluid due to heat and mass transfer through a porous medium bounded by an infinite vertical plate under the action of an external transfer magnetic field of uniform strength $B_{0}$. The fluid considered is a gray, absorbing-emitting radiation but nonscattering medium. It is assumed that there exists a homogeneous first-order chemical reaction between the fluid and species concentration. Then, by usual Boussinesq's approximation, the unsteady flow is governed by the following equations:

$$
\begin{gathered}
\frac{\partial v^{\prime}}{\partial y^{\prime}}=0 \\
\frac{\partial u^{\prime}}{\partial t^{\prime}}+v^{\prime} \frac{\partial u^{\prime}}{\partial y^{\prime}}=g \beta\left(T^{\prime}-T_{\infty}^{\prime}\right)+g \beta^{*}\left(C^{\prime}-C_{\infty}^{\prime}\right)+v \frac{\partial^{2} u^{\prime}}{\partial y^{\prime 2}}-\left(\frac{\sigma B_{o}^{2}}{\rho}+\frac{v}{K}\right) u^{\prime} \\
\frac{\partial T^{\prime}}{\partial t^{\prime}}+v^{\prime} \frac{\partial T^{\prime}}{\partial y^{\prime}}=\alpha \frac{\partial^{2} T^{\prime}}{\partial y^{\prime 2}}+Q\left(T^{\prime}-T_{\infty}^{\prime}\right)-\frac{1}{\rho c_{p}} \frac{\partial q_{r}^{*}}{\partial y^{\prime}} \\
\frac{\partial C^{\prime}}{\partial t^{\prime}}+v^{\prime} \frac{\partial C^{\prime}}{\partial y^{\prime}}=D \frac{\partial^{2} C^{\prime}}{\partial y^{\prime 2}}-R^{*}\left(C^{\prime}-C_{\infty}^{\prime}\right) .
\end{gathered}
$$

For constant and uniform suction, (2.1) integrates to

$$
v^{\prime}=-v_{0}
$$

where the negative sign indicates that suction is towards the plate. 
The initial and boundary conditions are

$$
\begin{gathered}
u^{\prime}=0, \quad T^{\prime}=T_{\infty}^{\prime}, \quad C^{\prime}=C_{\infty}^{\prime} \quad \text { for } t \leq 0, \\
u^{\prime}=0, \quad T^{\prime}=T_{w,}^{\prime} \quad C^{\prime}=C_{w}^{\prime}, \quad \text { for } y^{\prime}=0, t>0, \\
u^{\prime} \longrightarrow 0, \quad T^{\prime} \longrightarrow T_{\infty}^{\prime} \quad C^{\prime} \longrightarrow C_{\infty}^{\prime} \quad \text { for } y^{\prime} \longrightarrow \infty, t>0 .
\end{gathered}
$$

The radiative heat flux under term by using the Rosseland approximation is given by

$$
q_{r}^{*}=-\frac{4 \sigma^{*}}{3 k^{*}} \frac{\partial T^{\prime}}{\partial y^{\prime}}
$$

We assume that the temperature differences within the flow are sufficiently small such that $T^{\prime 4}$ may be expressed as a linear function of the temperature. This is accomplished by expanding $T^{\prime 4}$ in a Taylor series about $T_{\infty}^{\prime}$ and neglecting higher-order terms, thus $T^{\prime 4}$ can be expressed as

$$
T^{\prime 4} \cong 4 T_{\infty}^{\prime 3} T^{\prime}-3 T_{\infty}^{\prime 4}
$$

By using (2.7) and (2.8), (2.3) reduces to

$$
\frac{\partial T^{\prime}}{\partial t^{\prime}}=\alpha \frac{\partial^{2} T^{\prime}}{\partial y^{\prime 2}}+Q\left(T^{\prime}-T_{\infty}^{\prime}\right)+\frac{16 \sigma^{*} T_{\infty}^{\prime 3}}{3 k^{*} \rho c_{p}} \frac{\partial^{2} T^{\prime}}{\partial y^{\prime 2}}
$$

To present solutions which are independent of geometry of the flow regime, we introduce the dimensionless variable as follows:

$$
\begin{gathered}
u=\frac{u^{\prime}}{v_{o}}, \quad y=\frac{y^{\prime} v_{o}}{v}, \quad t=\frac{t^{\prime} v_{o}^{2}}{v}, \\
\theta=\frac{T^{\prime}-T_{\infty}^{\prime}}{T_{w}^{\prime}-T_{\infty}^{\prime}}, \quad \phi=\frac{C^{\prime}-C_{\infty}^{\prime}}{C_{w}^{\prime}-C_{\infty}^{\prime}} .
\end{gathered}
$$

Substituting from (2.10) into (2.2), (2.9), and (2.4), we obtain

$$
\begin{gathered}
\frac{\partial u}{\partial t}-\frac{\partial u}{\partial y}=\frac{\partial^{2} u}{\partial y^{2}}+\operatorname{Gr} \theta+\operatorname{Gm} \phi-\left(\frac{1}{k}+M\right) u, \\
\left(1+\frac{4 R}{3}\right) \frac{\partial^{2} \theta}{\partial y^{2}}-\operatorname{Pr}\left(\frac{\partial \theta}{\partial t}-\frac{\partial \theta}{\partial y}\right)+\operatorname{Pr} \eta \theta=0, \\
\frac{\partial^{2} \phi}{\partial y^{2}}-\operatorname{Sc}\left(\frac{\partial \phi}{\partial t}-\frac{\partial \phi}{\partial y}\right)-\operatorname{Sc} \delta \phi=0,
\end{gathered}
$$


where

$$
\begin{gathered}
G r=\frac{g \beta v\left(T_{w}^{\prime}-T_{\infty}^{\prime}\right)}{v_{o}^{3}}, \quad G m=\frac{g \beta^{*} v\left(C_{w}^{\prime}-C_{\infty}^{\prime}\right)}{v_{o}^{3}}, \quad \delta=\frac{R^{*} v}{v_{o}^{2}} \\
k=\frac{K v_{o}^{2}}{v^{2}}, \quad M=\frac{\sigma B_{o}^{2} v}{\rho v_{o}^{2}}, \quad S c=\frac{v}{D}, \quad \eta=\frac{v Q}{v_{o}^{2}} \\
\delta=\frac{R^{*} v}{v_{o}^{2}}, \quad \operatorname{Pr}=\frac{\rho c_{p} v}{k_{1}}=\frac{v}{\alpha}, \quad R=\frac{4 \sigma^{*} T_{\infty}^{\prime 3}}{k^{*} k_{1}}
\end{gathered}
$$

The initial and boundary conditions in nondimensional form are

$$
\begin{gathered}
u=0, \quad \theta=0, \quad \phi=0, \quad \forall y, t \leq 0, \\
u=0, \quad \theta=1, \quad \phi=1, \quad \text { at } y=0, t>0, \\
u \longrightarrow 0, \quad \theta \longrightarrow 0, \quad \phi \longrightarrow 0 \text { as } y \longrightarrow \infty, t>0 .
\end{gathered}
$$

All the physical variables are defined in the nomenclature. The solutions are obtained for hydrodynamic flow field in the presence of thermal radiation, chemical reaction and heat source/sink.

\section{Analytic Solution}

In order to obtain the solution of the present problem, we will use the Laplace transform technique.

Applying the Laplace transform to the system of (2.11), and the boundary conditions (2.13), we get

$$
\begin{gathered}
\frac{\partial^{2} \bar{\theta}}{\partial y^{2}}+F_{2} \frac{\partial \bar{\theta}}{\partial y}-F_{2}(s-\eta) \bar{\theta}=0, \\
\frac{\partial^{2} \bar{\phi}}{\partial y^{2}}+\operatorname{Sc} \frac{\partial \bar{\phi}}{\partial y}-\operatorname{Sc}(s+\delta) \bar{\phi}=0, \\
\frac{\partial^{2} \bar{u}}{\partial y^{2}}+\frac{\partial \bar{u}}{\partial y}-\left(s+M^{\prime}\right) \bar{u}+G r \bar{\theta}+G m \bar{\phi}=0,
\end{gathered}
$$

where,

$$
M^{\prime}=\frac{1}{k}+M, \quad F_{2}=\frac{\operatorname{Pr}}{(1+(4 / 3) R)}
$$


$s$ is Laplace transformation parameter, $\bar{u}, \bar{\theta}$, and $\bar{\phi}$ are Laplace transformation of $u, \theta$, and $\phi$, respectively,

$$
\begin{gathered}
\bar{u}=0, \quad \bar{\theta}=\bar{\phi}=\frac{1}{s} \quad \text { at } y=0, t>0, \\
\bar{u}=\bar{\theta}=\bar{\phi}=0, \quad \text { as } y \longrightarrow \infty, t>0 .
\end{gathered}
$$

Solving the system of (3.1)-(3.3), with the help of the result in (3.5), we get

$$
\begin{gathered}
\bar{\theta}(y, s)=\frac{1}{s} \operatorname{Exp}\left(\lambda_{\theta} y\right), \\
\bar{\phi}(y, s)=\frac{1}{s} \operatorname{Exp}\left(\lambda_{\phi} y\right), \\
\bar{u}(y, s)=\bar{u}_{1}(y, s)+\bar{u}_{2}(y, s)+\bar{u}_{3}(y, s)+\bar{u}_{4}(y, s),
\end{gathered}
$$

where

$$
\begin{gathered}
\bar{u}_{1}(y, s)=\frac{-G r}{s}\left[\frac{e^{\lambda_{\theta} y}}{\lambda_{\theta}^{2}+\lambda_{\theta}-B_{4}}\right]=\frac{-G r}{s} e^{\lambda_{\theta} y}\left[\frac{(\alpha s+\gamma)-\beta \sqrt{s+B_{1}^{2}}}{\alpha^{2}\left[\left((s+W)^{2}+Q^{2}\right)\right]},\right. \\
\bar{u}_{2}(y, s)=\frac{-G m}{s}\left[\frac{e^{\lambda_{\phi} y}}{\lambda_{\phi}^{2}+\lambda_{\phi}-B_{4}}\right]=\frac{-G m}{s} e^{\lambda_{\phi} y}\left[\frac{\left(\alpha_{1} s+\gamma_{1}\right)-\beta_{1} \sqrt{s+B_{2}^{2}}}{\alpha_{1}^{2}\left[\left(\left(s+W_{1}\right)^{2}+Q_{1}^{2}\right)\right]}\right] \\
\bar{u}_{3}(y, s)=\frac{G r}{s}\left[\frac{e^{\lambda_{u} y}}{\lambda_{\theta}^{2}+\lambda_{\theta}-B_{4}}\right]=\frac{G r}{s} e^{\lambda_{u} y}\left[\frac{(\alpha s+\gamma)-\beta \sqrt{s+B_{1}^{2}}}{\alpha^{2}\left[\left((s+W)^{2}+Q^{2}\right)\right]},\right. \\
\bar{u}_{4}(y, s)=\frac{G m}{s}\left[\frac{e^{\lambda_{u} y}}{\lambda_{\phi}^{2}+\lambda_{\phi}-B_{4}}\right]=\frac{G m}{s} e^{\lambda_{u} y}\left[\frac{\left(\alpha_{1} s+\gamma_{1}\right)-\beta_{1} \sqrt{s+B_{2}^{2}}}{\alpha_{1}^{2}\left[\left(\left(s+W_{1}\right)^{2}+Q_{1}^{2}\right)\right]}\right]
\end{gathered}
$$

where

$$
\begin{array}{r}
B_{1}^{2}=\frac{F_{2}}{4}-\eta, \quad \lambda_{\theta}=-\frac{F_{2}}{2}-\sqrt{F_{2}} \sqrt{s+B_{1}^{2}}, \quad B_{2}^{2}=\frac{\mathrm{Sc}}{4}+\delta, \\
\lambda_{\phi}=-\frac{\mathrm{Sc}}{2}-\sqrt{\mathrm{Sc}} \sqrt{s+B_{2}^{2}}, \quad B_{3}^{2}=\frac{1}{4}+M^{\prime}, \quad \lambda_{u}=-\frac{1}{2}-\sqrt{s+B_{3}^{2}}, \\
\alpha=F_{2}-1, \quad \beta=\alpha \sqrt{F_{2}}, \quad \gamma=\frac{F_{2}}{2} \alpha-F_{2} \eta-M^{\prime}, \quad W=\frac{2 \alpha \gamma-\beta^{2}}{2 \alpha^{2}},
\end{array}
$$




$$
\begin{aligned}
Q=\sqrt{\frac{\gamma^{2}-\beta^{2} B_{1}^{2}}{\alpha^{2}}-W^{2}}, \quad Q_{1}=\sqrt{\frac{\gamma_{1}^{2}-\beta_{1}^{2} B_{2}^{2}}{\alpha_{1}^{2}}-W_{1}^{2}}, \quad \alpha_{1}=\mathrm{Sc}-1, \\
\beta_{1}=\alpha_{1} \sqrt{\mathrm{Sc}}, \quad \gamma_{1}=\frac{\mathrm{Sc}^{2}}{2}+\mathrm{Sc}\left(\delta-\frac{1}{2}\right)-M^{\prime}, \quad W_{1}=\frac{2 \alpha_{1} \gamma_{1}-\beta_{1}^{2}}{2 \alpha_{1}^{2}}, \quad B_{4}=S+M^{\prime} .
\end{aligned}
$$

The inverse Laplace transformation of (3.6) is

$$
\begin{aligned}
\theta(y, t)=\frac{e^{-(y / 2) F_{2}}}{2}\{ & e^{y \sqrt{F_{2}} \sqrt{F_{2} / 4-\eta}} \operatorname{erf} c\left(\frac{y}{2} \sqrt{\frac{F_{2}}{t}}+B_{1} \sqrt{t}\right) \\
& \left.+e^{-y \sqrt{F_{2}} \sqrt{F_{2} / 4-\eta}} \operatorname{erf} c\left(\frac{y}{2} \sqrt{\frac{F_{2}}{t}}-B_{1} \sqrt{t}\right)\right\} .
\end{aligned}
$$

The analytic solution of (3.2) can be obtained by taking the inverse transforms of (3.7). So, the solution of the problem for the concentration $\phi(y, t)$ for $t>0$

$$
\phi(y, t)=\frac{e^{-(y / 2) S c}}{2}\left\{e^{y \sqrt{S c} B_{2}} \operatorname{erf} c\left(\frac{y}{2} \sqrt{\frac{S c}{t}}+B_{2} \sqrt{t}\right)+e^{-y \sqrt{S c} B_{2}} \operatorname{erf} c\left(\frac{y}{2} \sqrt{\frac{S c}{t}}-B_{2} \sqrt{t}\right)\right\}
$$

Similarly, the general solution of (3.3) can be obtained by taking the inverse Laplace transform of (3.8). The expressions for velocity, $u$, velocity gradient, $\partial u /\left.\partial y\right|_{y=0}$, temperature gradient, $\partial \theta /\left.\partial y\right|_{y=0}$ and concentration gradient, $\partial \phi /\left.\partial y\right|_{y=0}$ are shown in Appendix A.

\section{Numerical Results and Discussion}

In order to get physical insight into the problem, the numerical calculations are carried out to study the variations in velocity $u$, temperature $\theta$ and concentration $\phi$. The variation in skin-friction (shear stress at the wall), rate of heat and mass transfer are computed. These variations involve the effects of time $t$, heat generation parameter $\eta$, chemical reaction parameter $\delta$, Schmidt number Sc, Prandtl number Pr, thermal radiation parameter $R$, magnetic field parameter $M$ and permeability parameter $k$. The values of Prandtl number Pr are chosen to be 3, 7, and 10. The values of Schmidt number Sc are chosen to be 0.22, 0.62, and 0.78 , which represent hydrogen, water vapour and ammonia, respectively.

Representative velocity, temperature and concentration profiles across the boundary layer for different values of the dimensionless time $t$ are presented in Figure 1 . As the dimensionless time increases, the velocity, temperature and concentration profiles increase. 


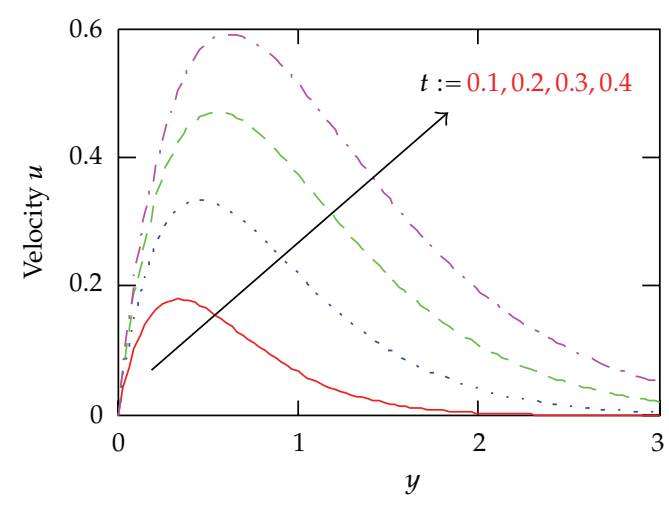

(a)

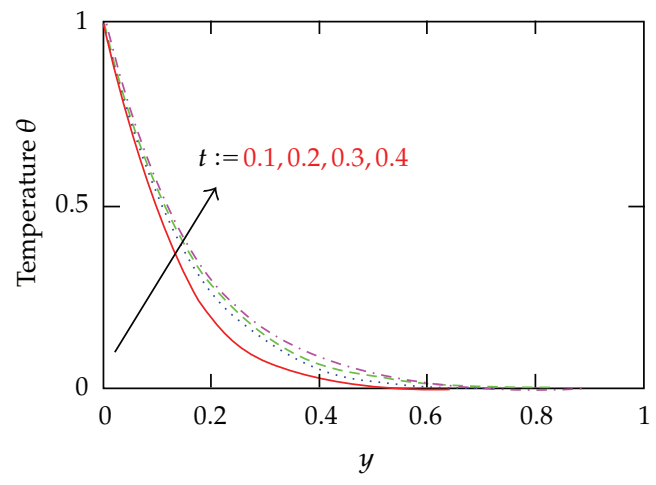

(b)

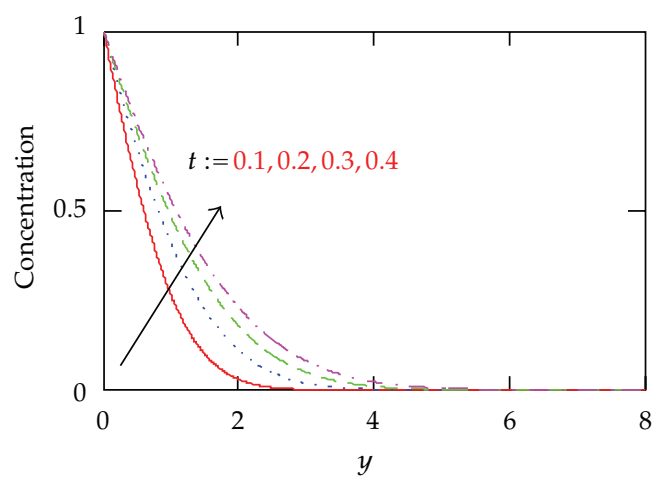

(c)

Figure 1: Velocity, temperature and concentration profiles against $y$ for various values of $t$ with $\operatorname{Pr}=10$, $M=0.2, \eta=-2, k=1, \mathrm{Sc}=0.22, G r=G m=5, \delta=0.1$, and $R=0.1$.

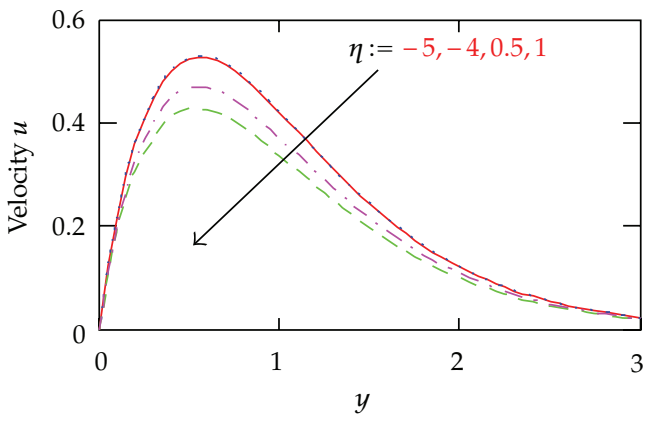

(a)

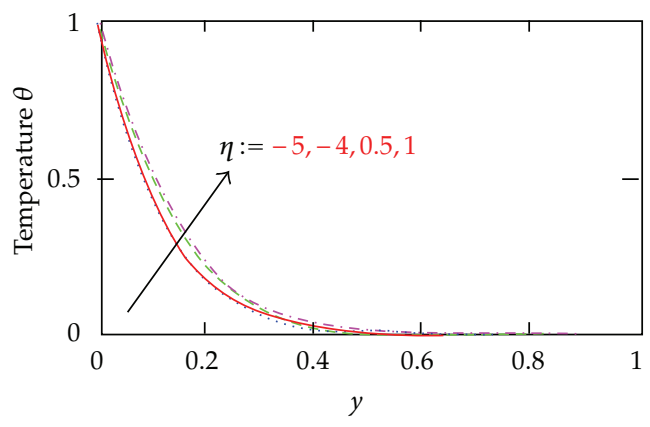

(b)

Figure 2: Velocity and temperature profiles against $y$ for various values of $\eta$ with $t=0.1, \operatorname{Pr}=10, M=0.2$, $k=1, \mathrm{Sc}=0.22, G r=G m=5, \delta=0.1$, and $R=0.1$.

Figure 2 describe the behavior of velocity and temperature profiles across the boundary layer for different values of the heat generation parameter $\eta$. As the heat source parameter $\eta$ increases, the velocity and temperature profiles increase. The volumetric heat source term may exert a strong influence on the heat transfer and as a consequence, also on the fluid flow. 


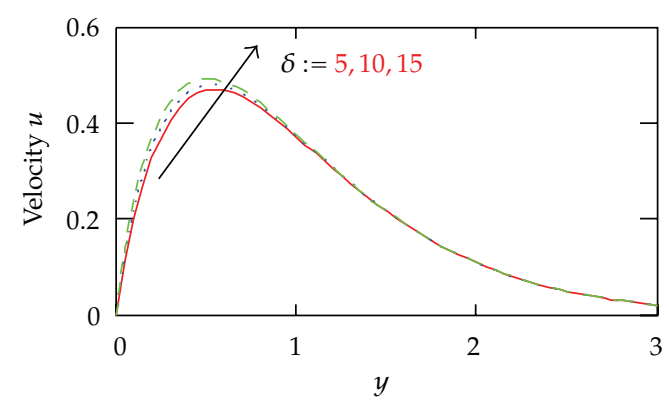

(a)

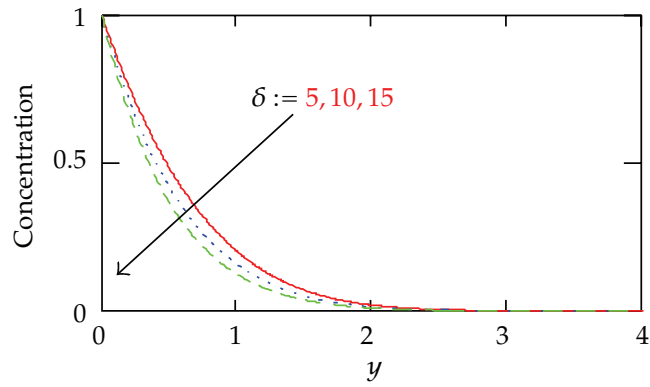

(b)

Figure 3: Velocity and concentration profiles against $y$ for various values of $\delta$ with $t=0.1, \operatorname{Pr}=10, M=0.2$, $k=1, \eta=-2, \mathrm{Sc}=0.22, \mathrm{Gr}=\mathrm{Gm}=5$, and $R=0.1$.

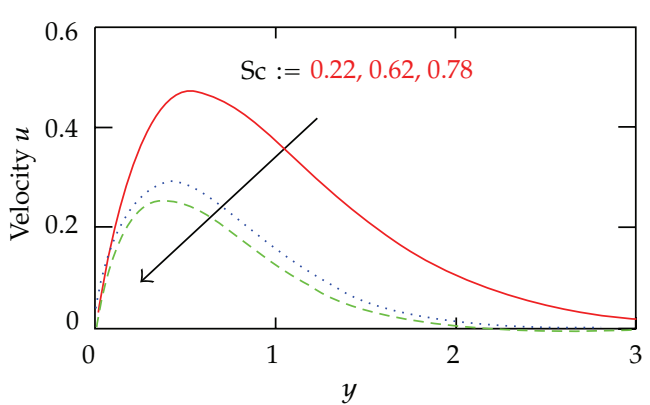

(a)

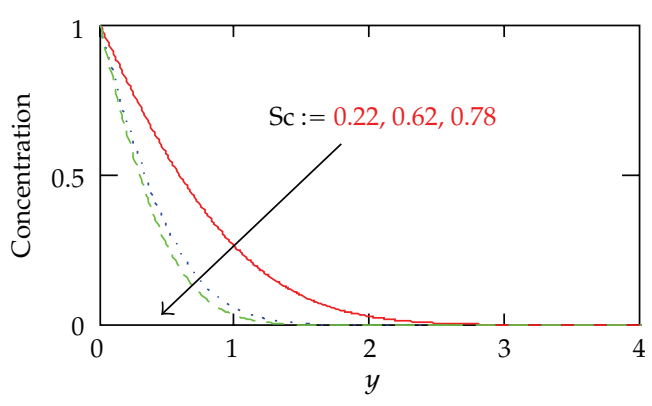

(b)

Figure 4: Velocity and concentration profiles against $y$ for various values of Sc with $t=0.1, \operatorname{Pr}=10$, $M=0.2, k=1, \eta=-2, G r=G m=5$, and $R=0.1$.

Figure 3 shows the velocity and concentration profiles for different values of chemical reaction parameter. As the chemical reaction parameter increases, the velocity increases but the concentration profile decreases.

Figure 4 displays the effects of Schmidt number on the velocity and concentration profiles, respectively. As the Schmidt number increases, the velocity and concentration decrease. Reductions in the velocity and concentration profiles are accompanied by simultaneous reductions in the velocity and concentration boundary layers. These behaviors are evident from Figure 4.

Figure 5 illustrates the velocity and temperature profiles for different values of Prandtl number. The numerical results show that the effect of increasing values of Prandtl number results in a decreasing velocity. Also, it is shown that an increase in the Prandtl number results tend to a decreasing of the thermal boundary layer and in general it lowers the average temperature through the boundary layer. The reason is that, the smaller values of $\operatorname{Pr}$ are equivalent to increase in the thermal conductivity of the fluid and therefore heat is able to diffuse away from the heated surface more rapidly for higher values of Pr. Hence in the case of smaller Prandtl numbers, the thermal boundary layer is thicker and the rate of heat transfer is reduced. 


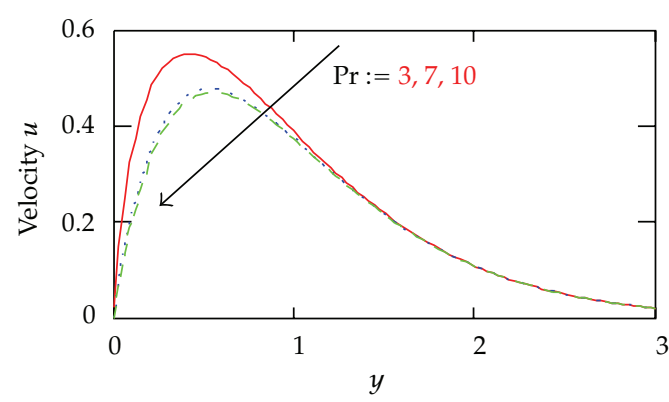

(a)

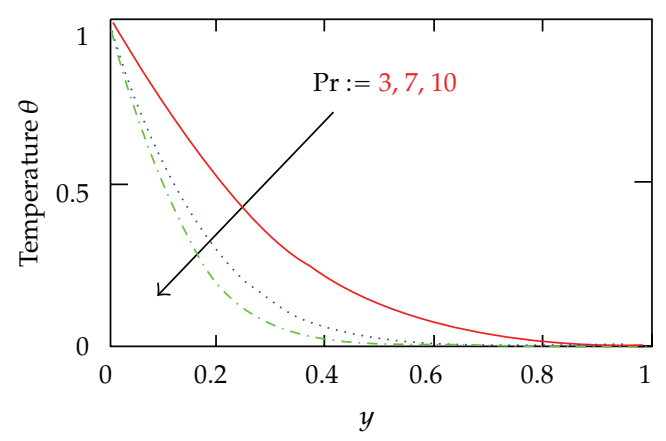

(b)

Figure 5: Velocity and temperature profiles against $y$ for various values of $\operatorname{Pr}$ with $t=0.1$, Sc $=0.22$, $M=0.2, k=1, \eta=-2, G r=G m=5$, and $R=0.1$.

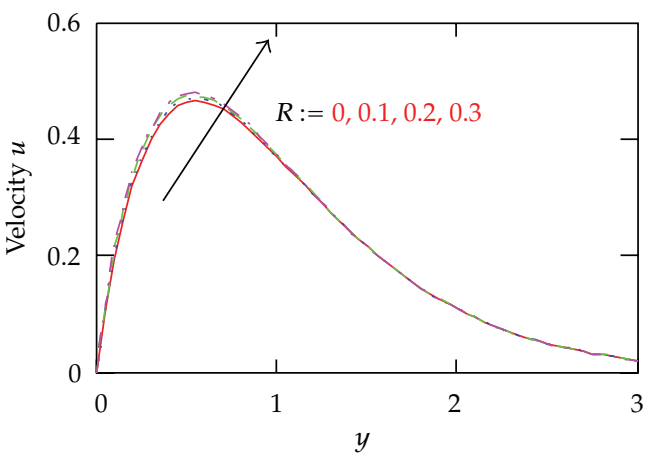

(a)

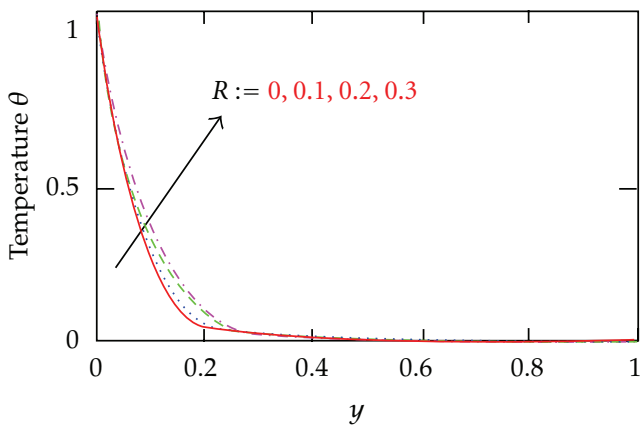

(b)

Figure 6: Velocity and temperature profiles against $y$ for various values of $R$ with $t=0.1, \operatorname{Pr}=10, M=0.2$, $k=1, \eta=-2, G r=G m=5.0$, and $\mathrm{Sc}=0.22$.

For different values of the radiation parameter $R$, the velocity and temperature profiles are shown in Figure 6. It is noticed that an increase in the radiation parameter results an increase in the velocity and temperature within the boundary layer, also it increases the thickness of the velocity and temperature boundary layers.

The velocity profiles for different values of the magnetic field parameter and dimensionless permeability are shown in Figure 7. It is clear that the velocity decreases with increasing of the magnetic field parameter. It is because that the application of transverse magnetic field will result a restrictive type force (Lorentz's force), similar to drag force which tends to restrictive the fluid flow and thus reducing its velocity. The presence of porous media increases the resistance flow resulting in a decrease in the flow velocity. This behavior is depicted by the decrease in the velocity as permeability decreases and when $k \rightarrow \infty$ (i.e., the porous medium effect is vanishes) the velocity is greater in the flow field. These behaviors are shown in Figure 7.

Figure 8 displays the effect of Sc, $t, M, k, \delta$, and Pr on shear stress $u^{\prime}(0, t)$ with respect to radiation parameter $R$, it is obvious that there is a slight changes in shear stress, also, it is 


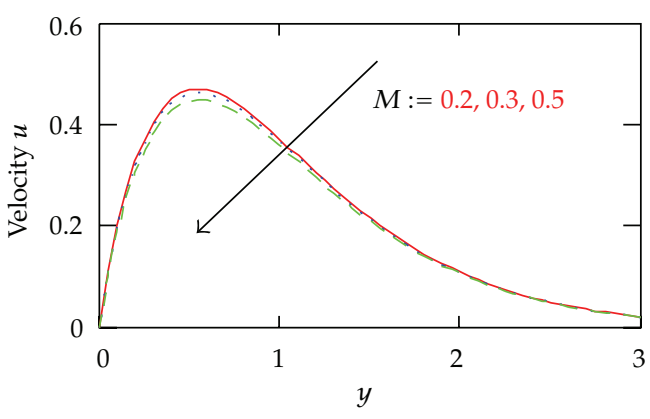

(a)

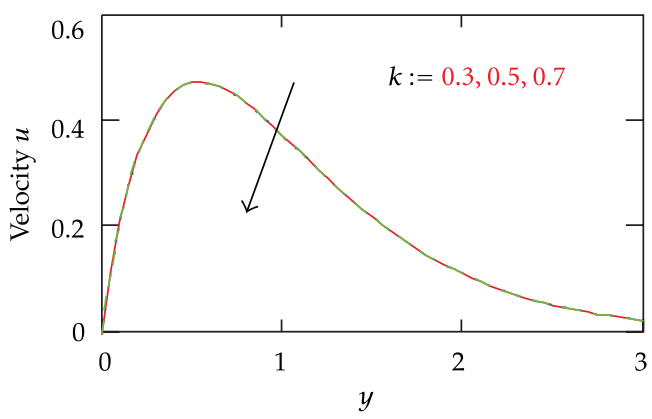

(b)

Figure 7: Velocity profiles against $y$ for various valuesof $M$ and $k$ with $t=0.1, \operatorname{Pr}=10, M=0.2, \mathrm{Sc}=0.22$, $R=0.1$, and $\eta=-2$.

seen that shear stress increases with an increasing of $k$ and $\delta$ but decreases with an increasing values of Sc, $t, M$, and Pr.

Figure 9 shows the influence of time, heat source parameter and the radiation parameter on the negative values of gradient temperature (i.e., $\left.-\theta^{\prime}(0, t)\right)$ with respect to the Prandtl number, it is seen that the increasing values of the time, heat source parameter and radiation parameter tend to decreasing in the negative values of the temperature gradient, also, it increases with the increasing of Pr.

Figure 10 displays the influence of time and chemical reaction parameter on the negative values of concentration gradient (i.e., $\left.-\phi^{\prime}(0, t)\right)$ respect the Schmidt number, it is concluded that it increases with the increasing of Sc and chemical reaction $\delta$ but decreases with an increase of $t$.

\section{Conclusion}

A mathematical model has been presented for analytically studies the thermal radiation and chemical reaction effect on unsteady MHD convection through a porous medium bounded by an infinite vertical plate. The fluid considered here is a gray, absorbing-emitting but nonscattering medium and the Rosseland approximation is used to describe the radiative heat flux in the energy equation. The dimensionless governing equations are solved using Laplace transform technique. The resulting velocity, temperature and concentration profiles as well as the skin-friction, rate of heat and mass transfer are shown graphically for different values of physical parameters involved. It has been shown that the fluid is accelerated, that is, velocity $(u)$ is increased with an increasing values of time $(t)$, chemical reaction parameter $(\delta)$, Prandtl number $(\operatorname{Pr})$, radiation parameter $(R)$, while they show opposite tends with an increasing values of heat source parameter $(\eta)$, Schmidt number (Sc) and magnetic field parameter $(M)$. Also, it is shown that velocity $(u)$ does not affected with the various values of the dimensionless permeability $k$. We conclude that, the negative temperature gradient $\left(-\theta^{\prime}(0, t)\right)$ increases as Pr increases but decreases as $t, \eta$, and $R$ increase. Finally, we obvious that, the negative concentration gradient $\left(-\phi^{\prime}(0, t)\right)$ increases as Sc and $\delta$ increases but decreases as $t$ increase.

It is hoped that the results obtained here will not only provide useful information for applications, but also serve as a complement to the previous studies. 


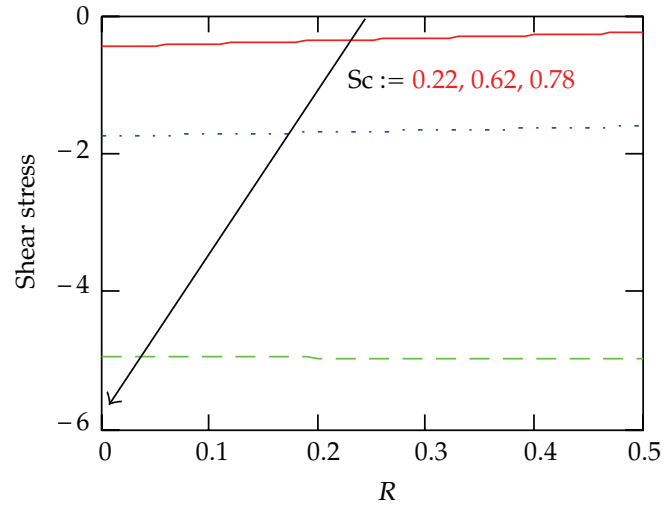

(a)

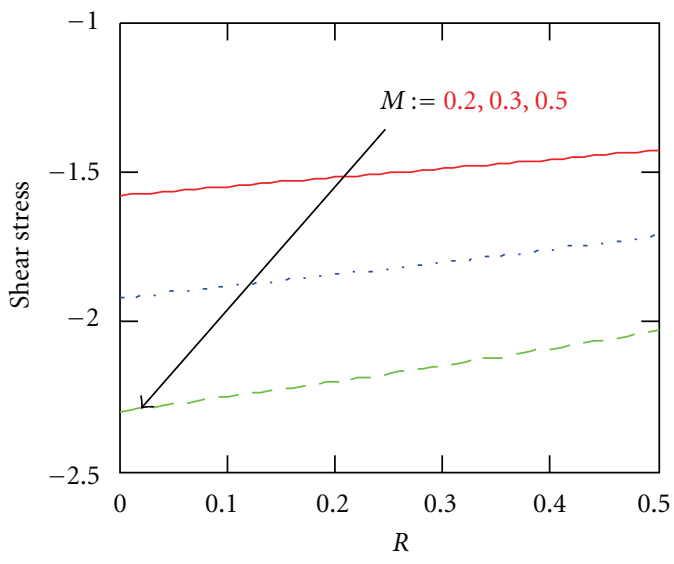

(c)

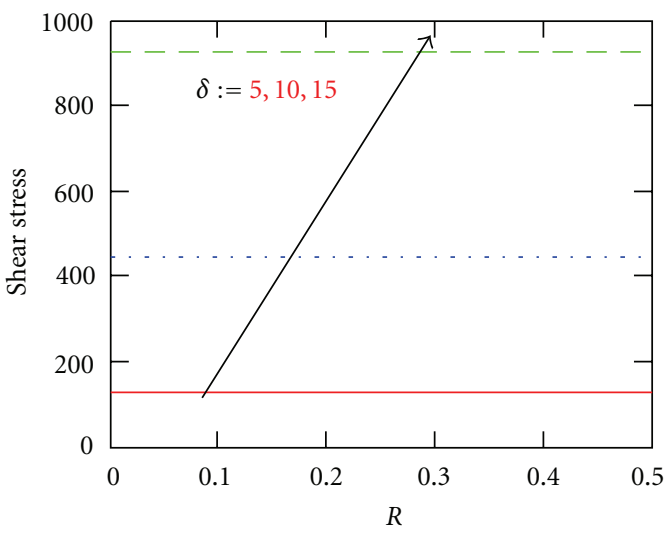

(e)

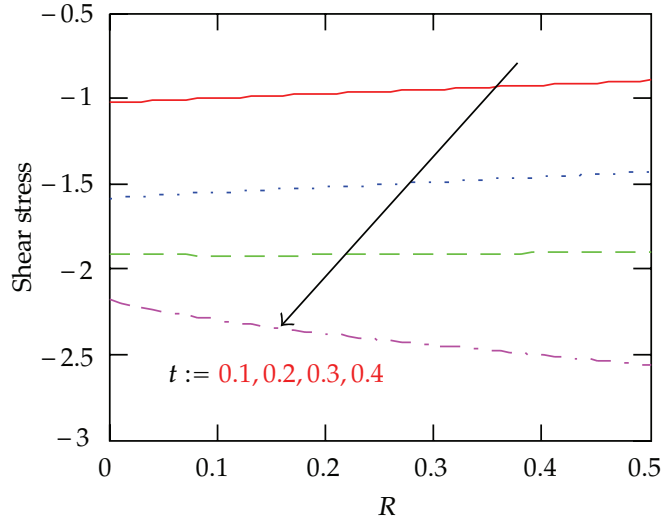

(b)

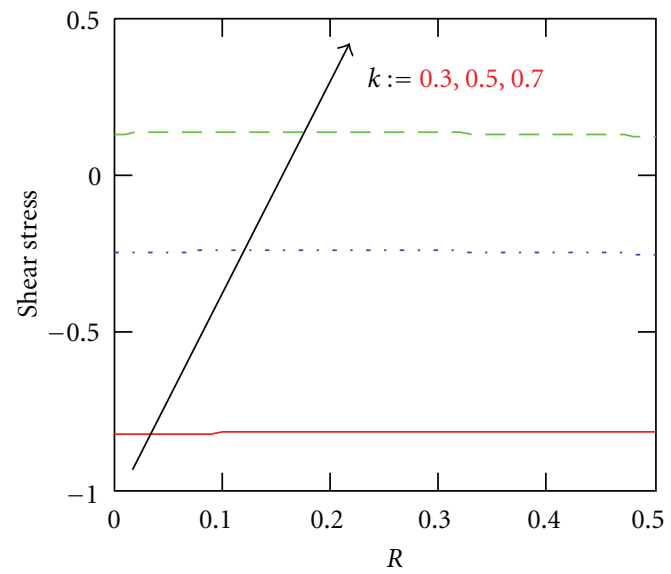

(d)

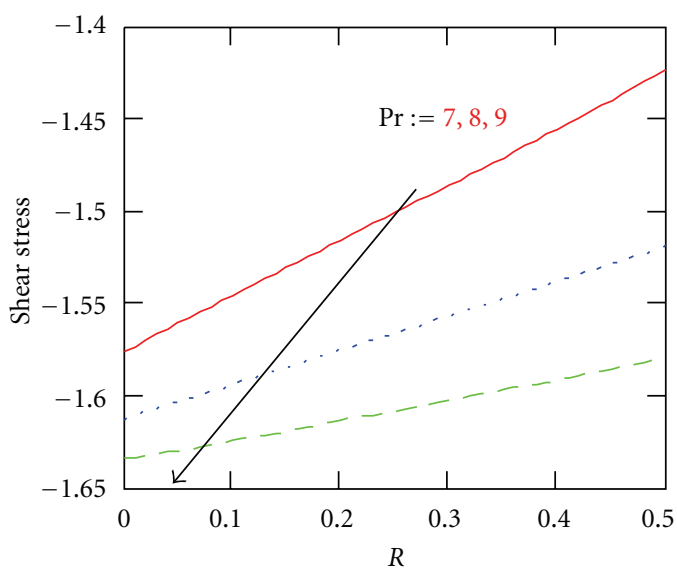

(f)

Figure 8: Variation of shear stress $u^{\prime}(0, t)$ against $R$ for various values of Sc, $t, M, k, \delta$, and Pr with constant $\operatorname{Pr}=10, M=0.2, k=1, \mathrm{Sc}=0.22, \mathrm{Gm}=\mathrm{Gr}=5$, and $\eta=-2$. 


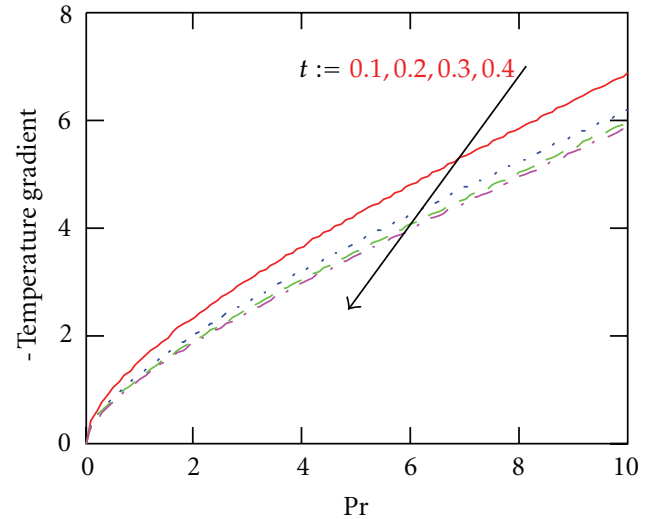

(a)

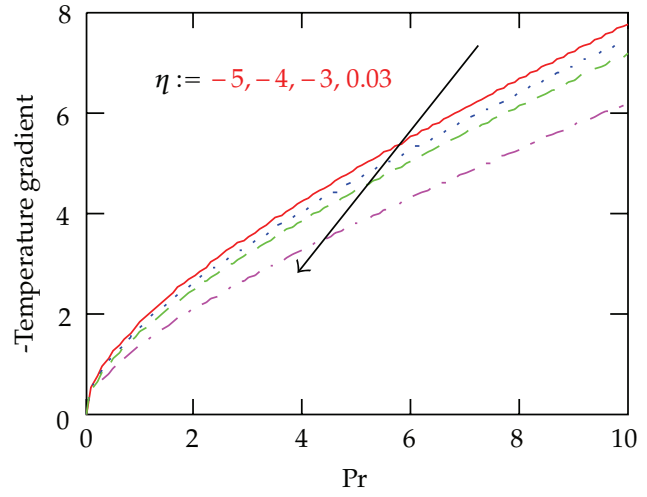

(b)

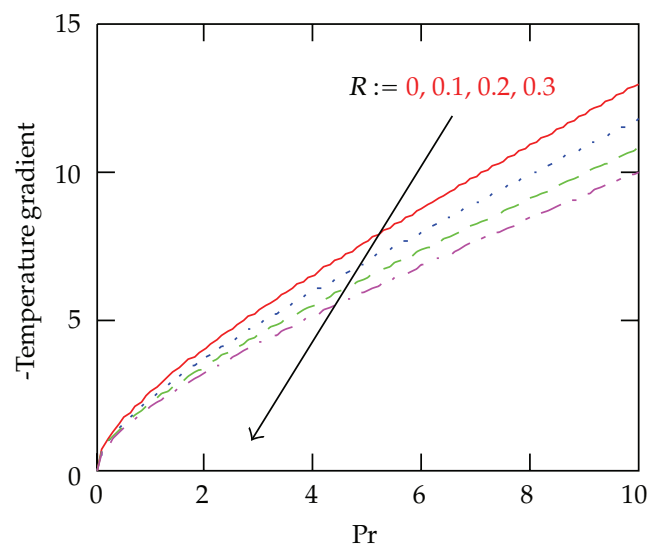

(c)

Figure 9: Variation of $-\theta^{\prime}(0, t)$ for various values of $t, \eta$, and $R$ with $\operatorname{Pr}=10, M=0.2, k=1, \mathrm{Sc}=0.22$, $G r=G m=5.0$, and $\eta=-2$.

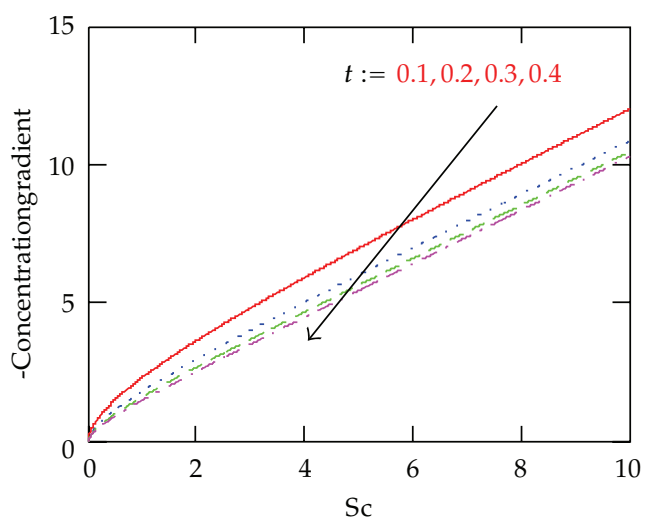

(a)

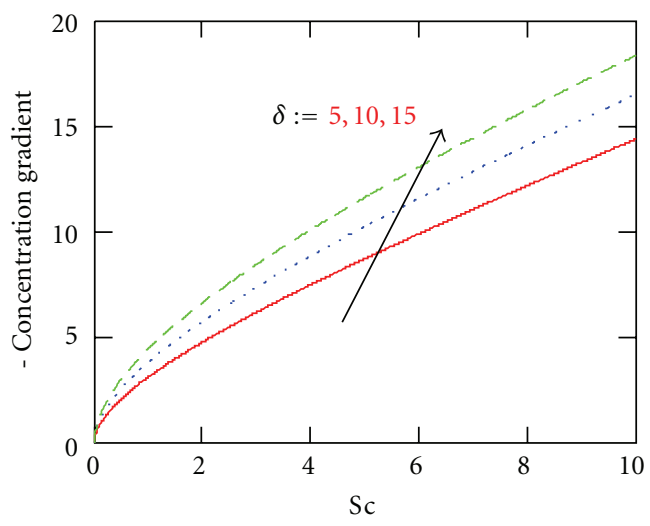

(b)

Figure 10: Variation of $-\phi^{\prime}(0, t)$ for various values of $t$ and $\delta$ with $\operatorname{Pr}=10, M=0.2, k=1, \mathrm{Sc}=0.22$, $G r=G m=5.0$, and $\eta=-2$. 
Mathematical Problems in Engineering

\section{Appendix}

A.

The inverse laplace transformation of (3.8) is

$$
u(y, t)=u_{1}(y, t)+u_{2}(y, t)+u_{3}(y, t)+u_{4}(y, t)
$$

where

$$
\begin{aligned}
& u_{1}(y, t)=-\frac{G r}{\alpha^{2}}\left\{\alpha \int_{0}^{t} \theta(y, u) e^{-W(t-u)} \cos Q(t-u) d u+\frac{r-\alpha W}{Q}\right. \\
& \times \int_{0}^{t} \theta(y, u) e^{-W(t-u)} \sin Q(t-u) d u-\beta \int_{0}^{t} \theta(y, u) G_{1}(t-u) d u \\
& -\beta\left(B_{1}^{2}-2 W\right) \int_{0}^{t} \theta(y, u) \int_{0}^{t-u} G_{1}(\tau) e^{-W(t-u-\tau)} \cos Q(t-u-\tau) d \tau d u \\
& +\frac{\beta}{Q}\left[W\left(B_{1}^{2}-2 W\right)+\left(W^{2}+Q^{2}\right)\right] \\
& \left.\times\left[\int_{0}^{t} \theta(y, u) \int_{0}^{t-u} G_{1}(\tau) e^{-W(t-u-\tau)} \sin Q(t-u-\tau) d \tau d u\right]\right\}, \\
& u_{2}(y, t)=-\frac{G m}{\alpha_{1}^{2}}\left\{\alpha_{1} \int_{0}^{t} \phi(y, u) e^{-W_{1}(t-u)} \cos Q_{1}(t-u) d u+\frac{\gamma_{1}-\alpha_{1} W_{1}}{Q_{1}}\right. \\
& \times \int_{0}^{t} \phi(y, u) e^{-W_{1}(t-u)} \sin Q_{1}(t-u) d u-\beta_{1} \int_{0}^{t} \phi(y, u) G_{2}(t-u) d u \\
& -\beta_{1}\left(B_{2}^{2}-2 W_{1}\right) \int_{0}^{t} \phi(y, u) \int_{0}^{t-u} G_{2}(\tau) e^{-W_{1}(t-u-\tau)} \cos Q_{1}(t-u-\tau) d \tau d u \\
& +\frac{\beta_{1}}{Q_{1}}\left[W_{1}\left(B_{2}^{2}-2 W_{1}\right)+\left(W_{1}^{2}+Q_{1}^{2}\right)\right] \int_{0}^{t} \phi(y, u) \\
& \left.\times \int_{0}^{t-u} G_{2}(\tau) e^{-W_{1}(t-u-\tau)} \sin Q_{1}(t-u-\tau) d \tau d u\right\} \\
& u_{3}(y, t)=\frac{G r}{\alpha^{2}}\left\{\alpha \int_{0}^{t} \theta_{1}(y, u) e^{-W(t-u)} \cos Q(t-u) d u+\frac{\gamma-\alpha W}{Q_{1}}\right. \\
& \times \int_{0}^{t} \theta_{1}(y, u) e^{-W(t-u)} \sin Q(t-u) d u-\beta \int_{0}^{t} \theta_{1}(y, u) G_{1}(t-u) d u \\
& -\beta\left(B_{1}^{2}-2 W\right) \int_{0}^{t} \theta_{1}(y, u) \int_{0}^{t-u} G_{1}(\tau) e^{-W(t-u-\tau)} \cos Q(t-u-\tau) d \tau d u
\end{aligned}
$$




$$
\begin{aligned}
&+ \frac{\beta}{Q}\left[W\left(B_{1}^{2}-2 W\right)+\left(W^{2}+Q^{2}\right)\right] \int_{0}^{t} \theta_{1}(y, u) \\
&\left.\times \int_{0}^{t-u} G_{1}(\tau) e^{-W(t-u-\tau)} \sin Q(t-u-\tau) d \tau d u\right\}, \\
& u_{4}(y, t)=\frac{G m}{\alpha_{1}^{2}}\left\{\alpha_{1} \int_{0}^{t} \theta_{1}(y, u) e^{-W_{1}(t-u)} \cos Q_{1}(t-u) d u+\frac{\gamma_{1}-\alpha_{1} W_{1}}{Q_{1}}\right. \\
& \times \int_{0}^{t} \theta_{1}(y, u) e^{-W_{1}(t-u)} \sin Q_{1}(t-u) d u-\beta_{1} \int_{0}^{t} \theta_{1}(y, u) G_{2}(t-u) d u \\
&-\beta_{1}\left(B_{2}^{2}-2 W_{1}\right) \int_{0}^{t} \theta_{1}(y, u) \int_{0}^{t-u} G_{2}(\tau) e^{-W_{1}(t-u-\tau)} \cos Q_{1}(t-u-\tau) d \tau d u \\
&+\frac{\beta_{1}}{Q_{1}}\left[W_{1}\left(B_{2}^{2}-2 W_{1}\right)+\left(W_{1}^{2}+Q_{1}^{2}\right)\right] \int_{0}^{t} \theta_{1}(y, u) \\
&\left.\times \int_{0}^{t-u} G_{2}(\tau) e^{-W_{1}(t-u-\tau)} \sin Q_{1}(t-u-\tau) d \tau d u\right\},
\end{aligned}
$$

where

$$
\begin{gathered}
G_{1}(t)=\frac{1}{B_{1}} \operatorname{erf}\left(B_{1} \sqrt{t}\right), \\
G_{2}(t)=\frac{1}{B_{2}} \operatorname{erf}\left(B_{2} \sqrt{t}\right), \\
\theta_{1}(y, t)=\frac{1}{2} e^{-y / 2}\left\{e^{y B_{3}} \operatorname{erf} c\left(\frac{y}{2 \sqrt{t}}+B_{3} \sqrt{t}\right)+e^{-y B_{3}} \operatorname{erf}\left(\frac{y}{2 \sqrt{t}}-B_{3} \sqrt{t}\right)\right\} . \\
\theta^{\prime}(0, t)=-\frac{F_{2}}{2}-B_{1} \sqrt{F_{2}} \operatorname{erf}\left(B_{1} \sqrt{t}\right)-\sqrt{\frac{F_{2}}{\pi t} e^{-B_{1}^{2} t},} \\
\phi^{\prime}(0, t)=-\frac{\mathrm{Sc}}{2}-B_{2} \sqrt{\mathrm{Sc}} \operatorname{erf}\left(B_{2} \sqrt{t}\right)-\sqrt{\frac{\mathrm{Sc}}{\pi t}} e^{-B_{2}^{2} t}, \\
\frac{\left.\partial u(y, t)\right|_{y=0}=\left.\sum_{i=1}^{4} \frac{\partial}{\partial y} u_{i}(y, t)\right|_{y=0^{\prime}} \quad i=1,2,3,4,}{\left.\frac{\partial u_{1}}{\partial y}\right|_{y=0}=-\frac{G r}{\alpha^{2}}\left\{\alpha \int_{0}^{\sqrt{t}} \theta^{\prime}(0, x) e^{-W\left(t-x^{2}\right)} \cos Q\left(t-x^{2}\right) d x+\frac{\gamma-\alpha W}{Q}\right.}
\end{gathered}
$$


Mathematical Problems in Engineering

$$
\begin{aligned}
& \times \int_{0}^{\sqrt{t}} \theta^{\prime}(0, x) e^{-W\left(t-x^{2}\right)} \sin Q\left(t-x^{2}\right) d x-\beta \int_{0}^{\sqrt{t}} \theta^{\prime}(0, x) G_{1}\left(t-x^{2}\right) d x \\
& -\beta\left(B_{1}^{2}-2 W\right) \int_{0}^{\sqrt{t}} \Theta^{\prime}(0, x) \int_{0}^{t-x^{2}} G_{1}(\tau) e^{-W\left(t-x^{2}-\tau\right)} \\
& \times \cos Q\left(t-x^{2}-\tau\right) d \tau d x \\
& +\frac{\beta}{Q}\left[W\left(B_{1}^{2}-2 W\right)+\left(W^{2}+Q^{2}\right)\right] \int_{0}^{\sqrt{t}} \theta^{\prime}(0, x) \\
& \left.\times \int_{0}^{t-x^{2}} G_{1}(\tau) e^{-W\left(t-x^{2}-\tau\right)} \sin Q\left(t-x^{2}-\tau\right) d \tau d x\right\} \\
& \left.\frac{\partial u_{2}}{\partial y}\right|_{y=0}=-\frac{G m}{\alpha_{1}^{2}}\left\{\alpha_{1} \int_{0}^{\sqrt{t}} \phi^{\prime}(0, x) e^{-W_{1}\left(t-x^{2}\right)} \cos Q_{1}\left(t-x^{2}\right) d x+\frac{\gamma_{1}-\alpha_{1} W_{1}}{Q_{1}}\right. \\
& \times \int_{0}^{\sqrt{t}} \phi^{\prime}(0, x) e^{-W_{1}\left(t-x^{2}\right)} \sin Q_{1}\left(t-x^{2}\right) d x-\beta_{1} \int_{0}^{\sqrt{t}} \phi^{\prime}(0, x) G_{2}\left(t-x^{2}\right) d x \\
& -\beta_{1}\left(B_{2}^{2}-2 W_{1}\right) \int_{0}^{\sqrt{t}} \phi^{\prime}(0, x) \int_{0}^{t-x^{2}} G_{2}(\tau) e^{-W_{1}\left(t-x^{2}-\tau\right)} \\
& \times \cos Q_{1}\left(t-x^{2}-\tau\right) d \tau d x \\
& +\frac{\beta_{1}}{Q_{1}}\left[W_{1}\left(B_{2}^{2}-2 W_{1}\right)+\left(W_{1}^{2}+Q_{1}^{2}\right)\right] \int_{0}^{\sqrt{t}} \phi^{\prime}(0, x) \\
& \left.\times \int_{0}^{t-x^{2}} G_{2}(\tau) e^{-W_{1}\left(t-x^{2}-\tau\right)} \sin Q_{1}\left(t-x^{2}-\tau\right) d \tau d x\right\} \\
& \left.\frac{\partial u_{3}}{\partial y}\right|_{y=0}=\frac{G r}{\alpha^{2}}\left\{\alpha \int_{0}^{\sqrt{t}} \theta_{1}^{\prime}(0, x) e^{-W\left(t-x^{2}\right)} \cos Q\left(t-x^{2}\right) d x+\frac{r-\alpha W}{Q}\right. \\
& \times \int_{0}^{\sqrt{t}} \theta^{\prime}(0, x) e^{-W\left(t-x^{2}\right)} \sin Q\left(t-x^{2}\right) d x-\beta \int_{0}^{\sqrt{t}} \theta^{\prime}(0, x) G_{1}\left(t-x^{2}\right) d x \\
& -\beta\left(B_{1}^{2}-2 W\right) \int_{0}^{\sqrt{t}} \theta^{\prime}(0, x) \int_{0}^{t-x^{2}} G_{1}(\tau) e^{-W\left(t-x^{2}-\tau\right)} \\
& \times \cos Q\left(t-x^{2}-\tau\right) d \tau d x \\
& +\frac{\beta}{Q}\left[W\left(B_{1}^{2}-2 W\right)+\left(W^{2}+Q^{2}\right)\right] \int_{0}^{\sqrt{t}} \theta^{\prime}(0, x) \\
& \left.\times \int_{0}^{t-x^{2}} G_{1}(\tau) e^{-W\left(t-x^{2}-\tau\right)} \sin Q\left(t-x^{2}-\tau\right) d \tau d x\right\}
\end{aligned}
$$




$$
\begin{aligned}
& \left.\frac{\partial u_{4}}{\partial y}\right|_{y=0}=\frac{G m}{\alpha_{1}^{2}}\left\{\alpha_{1} \int_{0}^{\sqrt{t}} \theta_{1}^{\prime}(0, x) e^{-W_{1}\left(t-x^{2}\right)} \cos Q_{1}\left(t-x^{2}\right) d x+\frac{\gamma_{1}-\alpha_{1} W_{1}}{Q_{1}}\right. \\
& \times \int_{0}^{\sqrt{t}} \theta_{1}^{\prime}(0, x) e^{-W_{1}\left(t-x^{2}\right)} \sin Q_{1}\left(t-x^{2}\right) d x-\beta_{1} \int_{0}^{\sqrt{t}} \theta_{1}^{\prime}(0, x) G_{2}\left(t-x^{2}\right) d x \\
& -\beta_{1}\left(B_{2}^{2}-2 W_{1}\right) \int_{0}^{\sqrt{t}} \theta_{1}^{\prime}(0, x) \int_{0}^{t-x^{2}} G_{2}(\tau) e^{-W_{1}\left(t-x^{2}-\tau\right)} \\
& \times \cos Q_{1}\left(t-x^{2}-\tau\right) d \tau d x \\
& +\frac{\beta_{1}}{Q_{1}}\left[W_{1}\left(B_{2}^{2}-2 W_{1}\right)+\left(W_{1}^{2}+Q_{1}^{2}\right)\right] \int_{0}^{\sqrt{t}} \theta_{1}^{\prime}(0, x) \\
& \left.\times \int_{0}^{t-x^{2}} G_{2}(\tau) e^{-W_{1}\left(t-x^{2}-\tau\right)} \sin Q_{1}\left(t-x^{2}-\tau\right) d \tau d x\right\} \\
& \theta^{\prime}(0, x)=\left.x \theta^{\prime}(0, t)\right|_{t=x^{2}}, \\
& \phi^{\prime}(0, x)=\left.x \phi^{\prime}(0, t)\right|_{t=x^{2}}, \\
& \Theta_{1}^{\prime}(0, t)=-\frac{1}{2}-B_{3} \operatorname{erf}\left(B_{3} \sqrt{t}\right)-\sqrt{\frac{1}{\pi t}} e^{-B_{3}^{2} t}, \\
& \Theta_{1}^{\prime}(0, x)=\left.x \Theta_{1}^{\prime}(0, t)\right|_{t=x^{2}} .
\end{aligned}
$$

\section{Nomenclatures}

\section{$B_{0}$ : Magnetic induction}

$C_{\infty}^{\prime}$ : Concentration of the fluid for away from the plate $C$ in the free stream

$C^{\prime}$ : Concentration of the fluid

$D$ : Chemical molecular diffusivity

g: Gravitational acceleration

$G_{m}$ : Solutal Grashof number

$G_{r}$ : Grashof number

$K$ : Permeability of the porous medium

$k$ : Dimensionless permeability

$k_{1}$ : Thermal conductivity of the fluid

$k^{*}$ : Mean absorption coefficient

M: Magnetic field parameter

Pr: Prandtl number

$q_{r}^{*}$ : Radiative heat flux

$Q:$ Hea tsource/sink coefficient

$R: \quad$ Radiation parameter

$R^{*}$ : First-order chemical reaction rate

Sc: Schmidt number

$t$ : Dimensionless time 


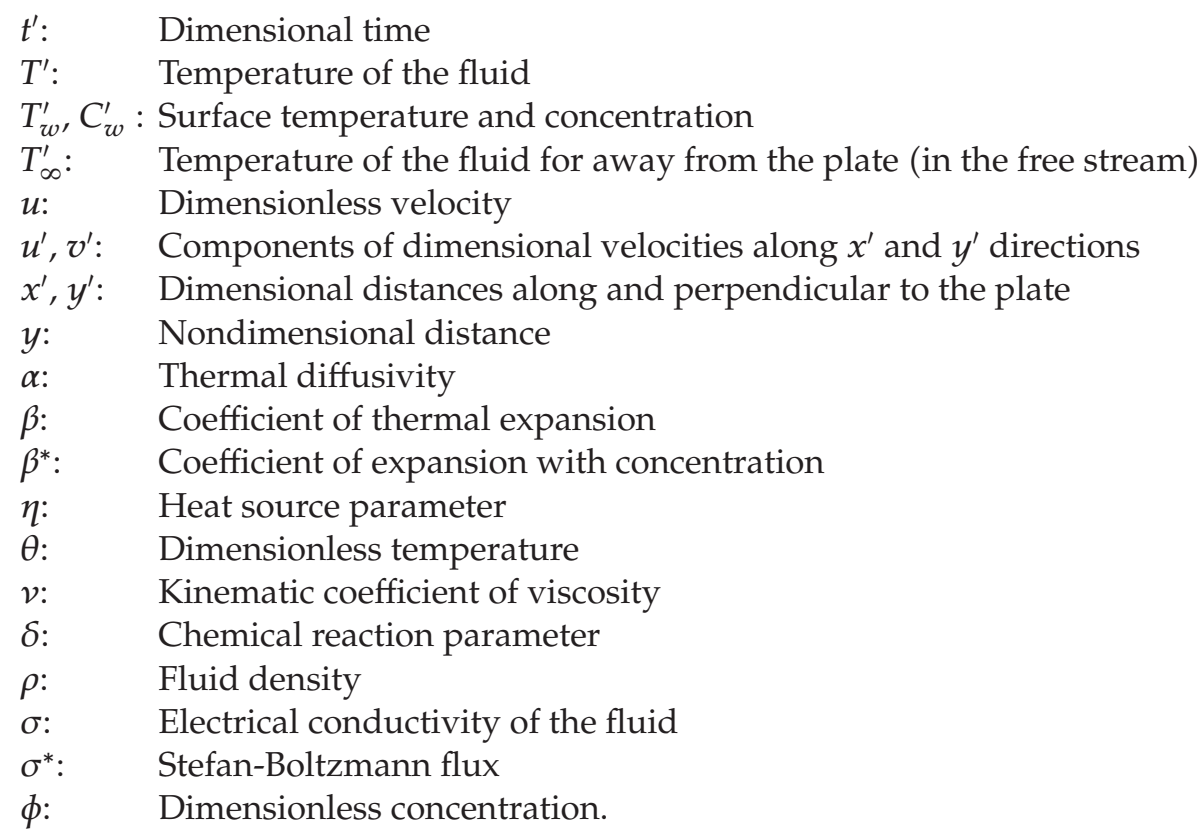

\section{References}

[1] M. M. Ali, T. S. Chen, and B. F. Armaly, "Natural convection-radiation interaction in boundary-layer flow over horizontal surfaces," AIAA Journal, vol. 22, no. 12, pp. 1797-1803, 1984.

[2] F. S. Ibrahim and F. M. Hady, "Mixed convection-radiation interaction in boundary-layer flow over horizontal surfaces," Astrophysics and Space Science, vol. 168, no. 2, pp. 263-276, 1990.

[3] M. A. Mansour, "Radiative and free-convection effects on the oscillatory flow past a vertical plate," Astrophysics and Space Science, vol. 166, no. 2, pp. 269-275, 1990.

[4] M. A. Hossain, M. A. Alim, and D. A. S. Rees, "The effect of radiation on free convection from a porous vertical plate," International Journal of Heat and Mass Transfer, vol. 42, no. 1, pp. 181-191, 1998.

[5] M. A. Hossain, K. Khanafer, and K. Vafai, "The effect of radiation on free convection flow of fluid with variable viscosity from a porous vertical plate," International Journal of Thermal Sciences, vol. 40, no. 2, pp. 115-124, 2001.

[6] A. Raptis and C. Perdikis, "Radiation and free convection flow past a moving plate," International Journal of Applied Mechanics and Engineering, vol. 4, no. 4, pp. 817-821, 1999.

[7] O. D. Makinde, "Free convection flow with thermal radiation and mass transfer past a moving vertical porous plate," International Communications in Heat and Mass Transfer, vol. 32, no. 10, pp. 1411-1419, 2005.

[8] M. D. Abdus-Sattar and M. D. Hamid Kalim, “Unsteady free-convection interaction with thermal radiation in a boundary-layer flow past a vertical porous plate," Journal of Mathematical and Physical Sciences, vol. 30, pp. 25-37, 1996.

[9] U. N. Das, R. Deka, and V. M. Soundalgekar, “Effects of mass transfer on flow past an impulsively started infinite vertical plate with constant heat flux and chemical reaction," Forschung im Ingenieurwesen, vol. 60, no. 10, pp. 284-287, 1994.

[10] A. J. Chamkha, "MHD flow of a uniformly streched vertical permeable surface in the presence of heat generation/absorption and a chemical reaction," International Communications in Heat and Mass Transfer, vol. 30, no. 3, pp. 413-422, 2003.

[11] R. Muthucumaraswamy and P. Ganesan, "On impulsive motion of a vertical plate with heat flux and diffusion of chemically reactive species," Forschung im Ingenieurwesen, vol. 66, no. 1, pp. 17-23, 2000.

[12] R. Muthucumaraswamy and P. Ganesan, "First-order chemical reaction on flow past an impulsively started vertical plate with uniform heat and mass flux," Acta Mechanica, vol. 147, no. 1-4, pp. 45-57, 2001. 
[13] R. Muthucumaraswamy, "Effects of suction on heat and mass transfer along a moving vertical surface in the presence of chemical reaction," Forschung im Ingenieurwesen, vol. 67, no. 3, pp. 129-132, 2002.

[14] A. Raptis and C. Perdikis, "Viscous flow over a non-linearly stretching sheet in the presence of a chemical reaction and magnetic field," International Journal of Non-Linear Mechanics, vol. 41, no. 4, pp. 527-529, 2006.

[15] M. A. Seddeek, A. A. Darwish, and M. S. Abdelmeguid, "Effects of chemical reaction and variable viscosity on hydromagnetic mixed convection heat and mass transfer for Hiemenz flow through porous media with radiation," Communications in Nonlinear Science \& Numerical Simulation, vol. 12, no. 2, pp. 195-213, 2007.

[16] F. S. Ibrahim, A. M. Elaiw, and A. A. Bakr, "Effect of the chemical reaction and radiation absorption on the unsteady MHD free convection flow past a semi infinite vertical permeable moving plate with heat source and suction," Communications in Nonlinear Science E Numerical Simulation, vol. 13, no. 6, pp. 1056-1066, 2008.

[17] A. Postelnicu, "Influence of chemical reaction on heat and mass transfer by natural convection from vertical surfaces in porous media considering Soret and Dufour effects," Heat and Mass Transfer, vol. 43, no. 6, pp. 595-602, 2007.

[18] R. A. Mohamed, I. A. Abbas, and S. M. Abo-Dahab, "Finite element analysis of hydromagnetic flow and heat transfer of a heat generation fluid over a surface embedded in a non-Darcian porous medium in the presence of chemical reaction," Communications in Nonlinear Science E Numerical Simulation, vol. 14, no. 4, pp. 1385-1395, 2009.

[19] R. A. Mohamed and S. M. Abo-Dahab, "Influence of chemical reaction and thermal radiation on the heat and mass transfer in MHD micropolar flow over a vertical moving porous plate in a porous medium with heat generation," International Journal of Thermal Sciences, vol. 48, no. 9, pp. 1800-1813, 2009. 


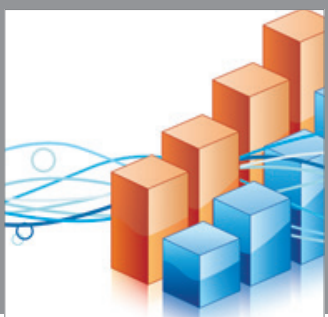

Advances in

Operations Research

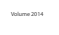

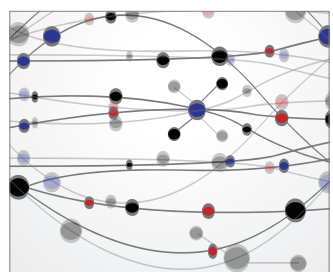

\section{The Scientific} World Journal
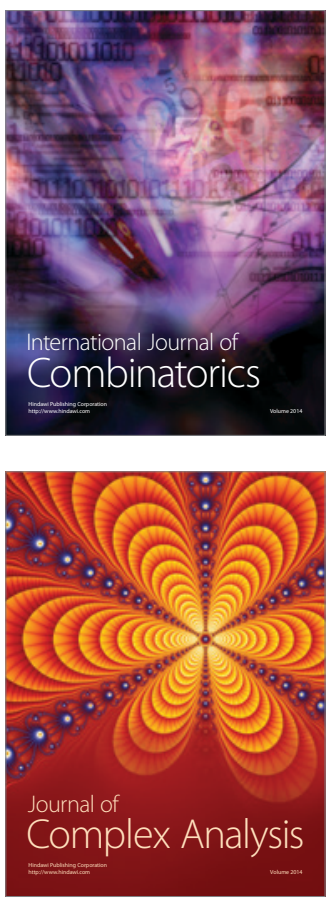

International Journal of

Mathematics and

Mathematical

Sciences
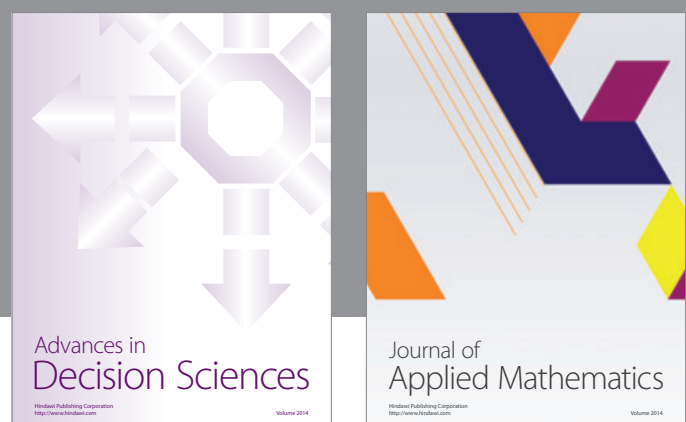

Journal of

Applied Mathematics
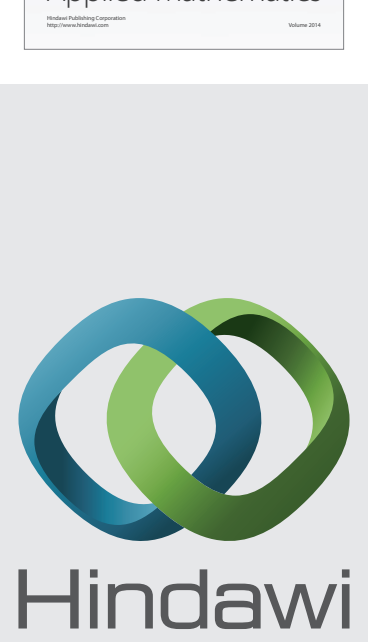

Submit your manuscripts at http://www.hindawi.com
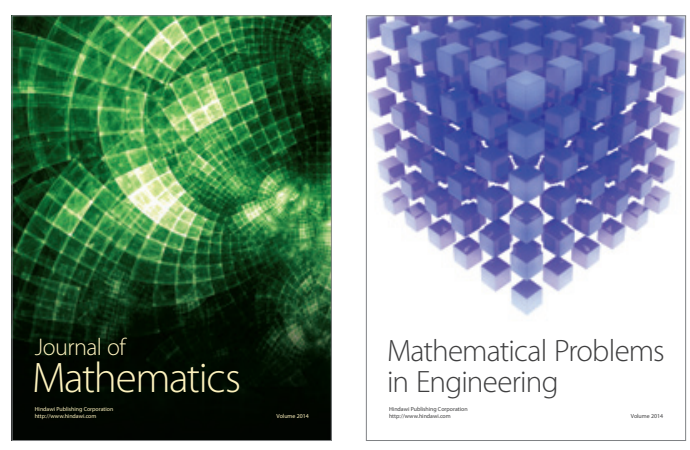

Mathematical Problems in Engineering
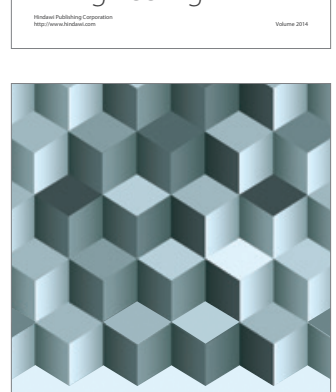

Journal of

Function Spaces
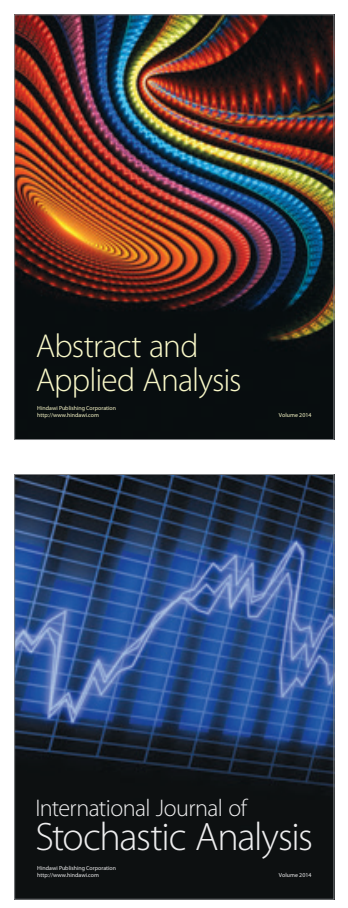

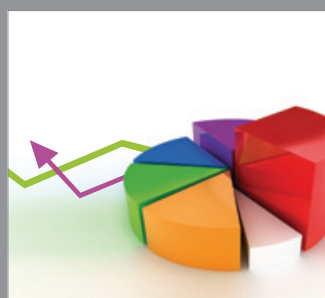

ournal of

Probability and Statistics

Promensencen
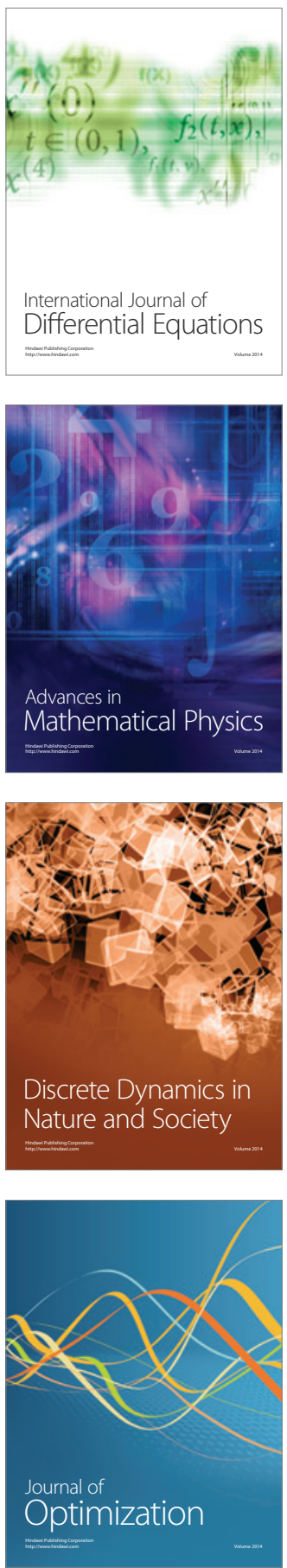